\title{
Retrieval of static stability parameter from the radiosonde/ rawinsonde ascent rate profiles: a wavelet approach
}

\author{
Prakash M. Dolas ${ }^{1, *}$ and Karanam Kishore Kumar ${ }^{1}$ \\ ${ }^{1}$ Space Physics Laboratory, Vikram sarabhai space center, Thiruvananthapuram-695022, India \\ * now at: Rajiv Gandhi Science and Technology Commission, Govt. of Maharashtra, India
}

Received: 17 September 2007 - Revised: 20 November 2008 - Accepted: 9 December 2008 - Published: 2 February 2009

\begin{abstract}
In the present communication a novel method is presented to derive the altitude profile of Brunt-Väisälä period from the ascent rate profile of sounding balloons. The basic premise of the present method is that the oscillations in the ascent rate of the balloon will have the signature of Brunt-Väisälä frequency, which can be retrieved by using sophisticated spectral tools. We employ wavelet transforms to arrive at the Brunt-Väisälä period profile. Comparison of retrieved Brunt-Väisälä periods with the values derived from the temperature data available from the same radiosonde ascent shows good agreement. Retrieving the atmospheric temperature from the height profile of Brunt-Väisälä period is also discussed in the present communication. We have shown that it is possible to estimate the Brunt-Väisälä period and temperature profiles from the rawinsonde ascent rate data alone where temperature sounding is not available.
\end{abstract}

Keywords. Meteorology and atmospheric dynamics (Mesoscale meteorology; Tropical meteorology; Instruments and techniques)

\section{Introduction}

Waves and oscillations in Earth's atmosphere, from shortperiod gravity waves to planetary scale, play a crucial role in several dynamical processes. Most importantly, these waves and oscillations couple the Earth's atmosphere vertically as well as horizontally, thus making the Earth's atmosphere as a single entity. The static stability of the atmosphere is an important prerequisite for atmospheric waves such as gravity waves to propagate vertically. The buoyancy oscillation in a stably stratified fluid, at the Brunt-Väisälä frequency $(N)$,

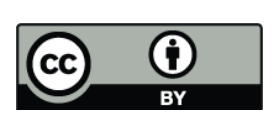

Correspondence to:

Karanam Kishore Kumar

(kishore_nmrf@yahoo.com) is a measure of the static stability of the medium. We shall use Brunt-Väisälä period $(1 / N)$ as well as Brunt-Väisälä frequency in this paper. In the atmosphere, it is estimated from the potential temperature gradient as

$N^{2}=\frac{g}{\theta} \frac{d \theta}{d z}$

where $g, \theta$ and $z$ are the acceleration due to gravity, potential temperature and height respectively. Alternately, $\mathrm{N}^{2}$ can also be expressed in terms of temperature gradient and dry adiabatic lapse rate $\Gamma_{d}$ as

$N^{2}=\frac{g}{T}\left[\frac{d T}{d z}-\Gamma_{d}\right]$

Positive values of $\mathrm{N}^{2}$ represent statically stable atmosphere whereas negative values represent the unstable state. Readers are referred to Holton (1992) or Salby (1996) for complete derivation and relevant discussion. There are several ways to determine this stability parameter. The required temperature and pressure profiles are readily provided by observational platforms such as a radiosonde. A novel approach has been reported in the past to obtain the height profile of $\mathrm{N}^{2}$ from mesosphere-stratosphere-troposphere (MST) radars (Rottger, 1980). High-resolution vertical air velocity spectra show a peak near N, what is usually referred to as "BruntVäisälä cut-off”. Once the Brunt-Väisälä frequency profile is obtained, the atmospheric temperature profile can easily be retrieved (Rottger, 1986; Revathy et al., 1996). However, this method has its own limitations in identifying the Brunt-Väisälä frequency, say, for example, when the horizontal wind velocity is high (Ecklund et al., 1985) of the order of $30 \mathrm{~ms}^{-1}$ and above. Recently, Hooper et al. (2004) carried out seminal work to retrieve the static stability parameter from MST radar return signal power observations. The authors exploit a well-known relationship between the radar return signal power (for a vertically directed beam) and the mean vertical gradient of generalized potential refractive

Published by Copernicus Publications on behalf of the European Geosciences Union. 
index to retrieve $\mathrm{N}^{2}$ profiles from VHF radar observations of the lower atmosphere.

Radiosonde measurements dominate over other observational techniques in the troposphere and lower stratosphere as they routinely measure atmospheric temperature, humidity and the horizontal wind vector, generally at predetermined pressure levels. The numerical weather prediction models are still primarily initialized by the radiosonde observations based analyses across the globe. Apart from routine soundings, radiosonde data have been used for studying the atmospheric oscillations caused by the gravity waves in the troposphere and lower stratosphere (e.g. Murrow and Henry, 1965; De Mandel and Scoggins, 1967; Weinstein et al., 1966; Massman, 1978; Vincent and Alexander, 2000; Wang and Geller, 2003). Jimspheres, constant-volume super-pressure balloons having conical projections on the exterior surface and made from aluminised mylar-sheet, are well suited for studying the atmospheric oscillations. Jimsphere retains its shape while minimizing the variation of aerodynamic drag as it ascends and maintains a nearly steady rate of ascent near $5 \mathrm{~ms}^{-1}$. Jimspheres have been used to derive the vertical air velocity from the ascent rate variations of the balloon with some limitations (De Mandel and Krivo, 1971). These limitations are mainly attributed to the balloon response to the varying buoyant and aerodynamic drag forces. On the other hand, routine radiosonde/rawinsonde balloons do not retain their spherical shape. They become oblate, thus changing the drag profile, and they also lose gas during ascends. There are several other factors related to the balloon and its motion that include aerodynamically induced balloon motions, icing, condensation, skin conductivity, and so on. All these contribute to the variations in the ascent rate of the balloon. However, the variations in the ascent rate can be used to study the atmospheric oscillations as these variations are large in comparison to balloon-induced variations (Massman, 1978).

Balloon's ascent rates have been used to study the atmospheric waves for the past several years. The first to use balloon's ascent rate to trace the atmospheric waves is Corby (1957). This technique is still used to explore the atmospheric waves (McHugh et al., 2008). In a seminal work, Reid (1972) compared the oscillations in ascent rate to measurements of the local air velocity made using radar, and showed that the oscillations in ascent rate correlate very well to the local air velocity. It was an important result, which has provided further credence for utilization of ascent rates to extract the atmospheric wave parameters. In another study Lalas and Einaudi (1980) estimated the vertical velocity due to waves using the measurements of horizontal velocity, and compared this with the measured fluctuations in the ascent rate of the balloon. The results showed a similar magnitude for fluctuations in the vertical velocity. In a very recent study, McHugh et al. (2008) emphatically showed the signature of mountain waves in the ascent rates. Apart from these studies, Kitchen and Shutts (1990) also used variations in the ascent rate of the balloon to study the gravity waves in the lower and middle stratosphere. They have shown that ascent rate of the balloon responds to the vertical motion of gravity waves generated in the vicinity of local mountains. One of the results reported by Kitchen and Shutts (1990) is the negative correlation between the rate of ascent and static stability profile of the radiosondes. Further, the authors report that the wave period experienced by the ascending balloon is often close to the average Brunt-Väisälä period in the layer in which waves were observed. All the above-mentioned studies essentially confirm that the balloon ascent rate does respond to the atmospheric oscillation; also that the gravity wave induced vertical motions are present and can be discerned in the variations of the ascent rates of rawinsonde balloons. The effect of these waves on the horizontal wind and temperature measurements that the radiosonde balloons provide are more difficult to isolate (Kitchen and Shutts, 1990). Thus studies carried out by Corby (1957), Reid (1972), Lalas and Einaudi (1980), Kitchen and Shutts (1990) and McHugh et al. (2008) beyond any doubt showed that the ascent rate of the balloon can be used to trace the atmospheric oscillations. The results reported in these studies form the basis for the present study.

Proceeding on similar lines, if the balloon as an ascending body immersed in a stratified medium responds to atmospheric oscillations then it may also respond to the natural oscillation in the atmosphere at the Brunt-Väisälä frequency. The central objective of the present study is to estimate the height profile of Brunt-Väisälä period in the troposphere and lower stratosphere from the ascent rate variations of radiosonde/rawinsonde balloons. By using the sophisticated spectral analysis tools it is possible to extract the time history of oscillations in the frequency domain. The present approach is a novel one in the sense that it uses wavelet analysis to extract the dominant oscillation in the ascent rate at any given height, which in turn is used to estimate BruntVäisälä period. The main focus of the present study is to extract the Brunt-Väisälä periods from the ascent rate profiles of the sounding balloons. An attempt is also made to compute the temperature profile by integrating Eq. (2) by using well-established inversion techniques. In Rawinsonde observations, where temperature measurements are not available, the present study finds its application. Section 2 provides the details of data, while Sect. 3 provides the description of methodology. Results are discussed in Sect. 4 and summary is presented in Sect. 5.

\section{Data}

Radiosonde observations from Chennai $\left(13.1^{\circ} \mathrm{N}, 80.4^{\circ} \mathrm{E}\right)$, India and rawinsonde observations from Shriharikota which is $100 \mathrm{~km}$ to the North of Chennai are used for the present study. The Chennai radiosonde data is provided by India meteorological Department (IMD) whereas Shriharikota rawinsonde data is provided by meteorological facility of Indian space research organization. The results presented here 
pertain to the period of 2-12 September 2002. The radiosondes are released twice daily at 00:00 and 12:00 UT at Chennai. For the present study we use 12:00 UT data from Chennai. However, at Shriharikota the rawinsonde observations were carried out only once at 09:00 UT. One-minute resolution ( $\sim 300-500 \mathrm{~m}$ height resolution) data from Chennai and $300 \mathrm{~m}$ data from Shriharikota (analyzed from $10 \mathrm{~Hz}$ records) are available. Chennai radiosonde data have height profiles of pressure, temperature, humidity and horizontal winds whereas only horizontal wind data are available from Shriharikota rawinsonde. An inter-comparison study of USA and Indian (IMD MKIII (mark three)) radiosonde data has shown that up to $100 \mathrm{mb}$ level, for $1 \mathrm{~min}$ interval data of IMD radiosonde, the mean difference is $<1 \mathrm{~K}$ and the precision determined for IMD radiosonde is $0.66 \mathrm{~K}$ for temperature (Schmidlin, 1988; Chakrabarty et al., 2000).

The ascent rate of the balloon, which is retrieved directly from the radar tracking, forms the basis for the present study. On most of the days the balloon reached up to $\sim 20 \mathrm{~km}$ in altitude and on some occasions up to $25 \mathrm{~km}$ and above. From the ascent rate of the balloon, the height traversed every minute is computed and this time series data are subjected to wavelet analysis to extract the dominant oscillations in the balloon motion. We now proceed to provide a brief description of wavelets used in the present study.

\section{Methodology: wavelet analysis}

From an historical point of view, wavelet analysis is a relatively new method, though its mathematical underpinnings date back all the way to the work of Joseph Fourier in the nineteenth century. A wavelet is a waveform of effectively limited duration that has an average value of zero. Wavelet analysis performs the breaking up of a signal into shifted and scaled versions of the original (or the mother) wavelet. One major advantage offered by wavelets is the ability to perform local analysis, that is, to analyze a localized area of a given signal. One further advantage of wavelet analysis is to provide the time history of the frequency content. This is very useful in analyzing the signal, whose frequency changes with time. By decomposing a time series into timefrequency space, one is able to determine both the dominant modes of variability and how those modes vary in time. The wavelet transform has been used for numerous studies in geophysics. A comprehensive description of geophysical applications can be found in Foufoula-Georgiou and Kumar (1995), while a theoretical treatment of wavelet analysis is given in Daubechies (1992). One criticism of wavelet analysis is the arbitrary choice of the wavelet function. In choosing the wavelet function several factors need to be considered (for discussion, refer to Farge, 1992).

The Wavelet Transform provides a time-frequency representation of the signal. The Wavelet Transform uses multiresolution technique by which different frequencies are an-

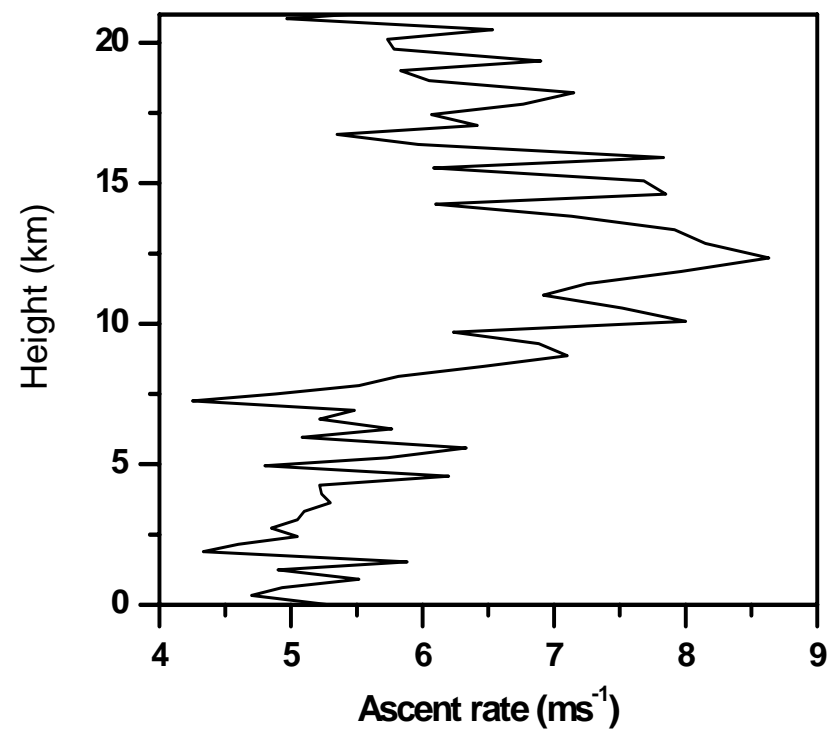

Fig. 1. Height profiles of balloon ascent rate on 2 September 2002.

alyzed with different resolutions. The Continuous Wavelet Transform (CWT) is provided by the following equation,

$X_{W T}(\tau, s)=\frac{1}{\sqrt{|s|}} \int x(t) \cdot \psi^{*}\left(\frac{t-\tau}{s}\right) d t$

where $x(t)$ is the signal to be analyzed. $\psi(t)$ is the mother wavelet or the basis function. All the wavelet functions used in the transformation are derived from the mother wavelet through translation (shifting) and scaling (dilation or compression). By now, many different types of mother wavelets are available to analyze a signal. The choice of the mother wavelet is very important for these analyses. The more appropriate choice is often the result of a trial and error process of determining which wavelet allows the most efficient extraction of the desired information. The Brunt-Väisälä oscillation of the atmosphere is a smoothly varying sinusoidal variation as a function of altitude. We know that Daubechies wavelet, because of its smoothness, provides a good match to the signal in the present study. By trial and error method it is decided to use order $10(\mathrm{db} 10)$ for the Daubechies wavelet as it was yielding the better results. However, there is subjectivity in choosing the order of the wavelet. The mother wavelet we chose provided the best discrimination between the Brunt-Väisälä oscillation and other components of the signal. These wavelets have no explicit expression except for $d b 1$, which is the Haar wavelet. For a detailed discussion on Daubechies wavelet, readers are refereed to Daubechies (1992).

\section{Results and discussion}

To demonstrate the method, we focus on 2 September 2002 radiosonde ascent from Chennai. Figure 1 shows the height 

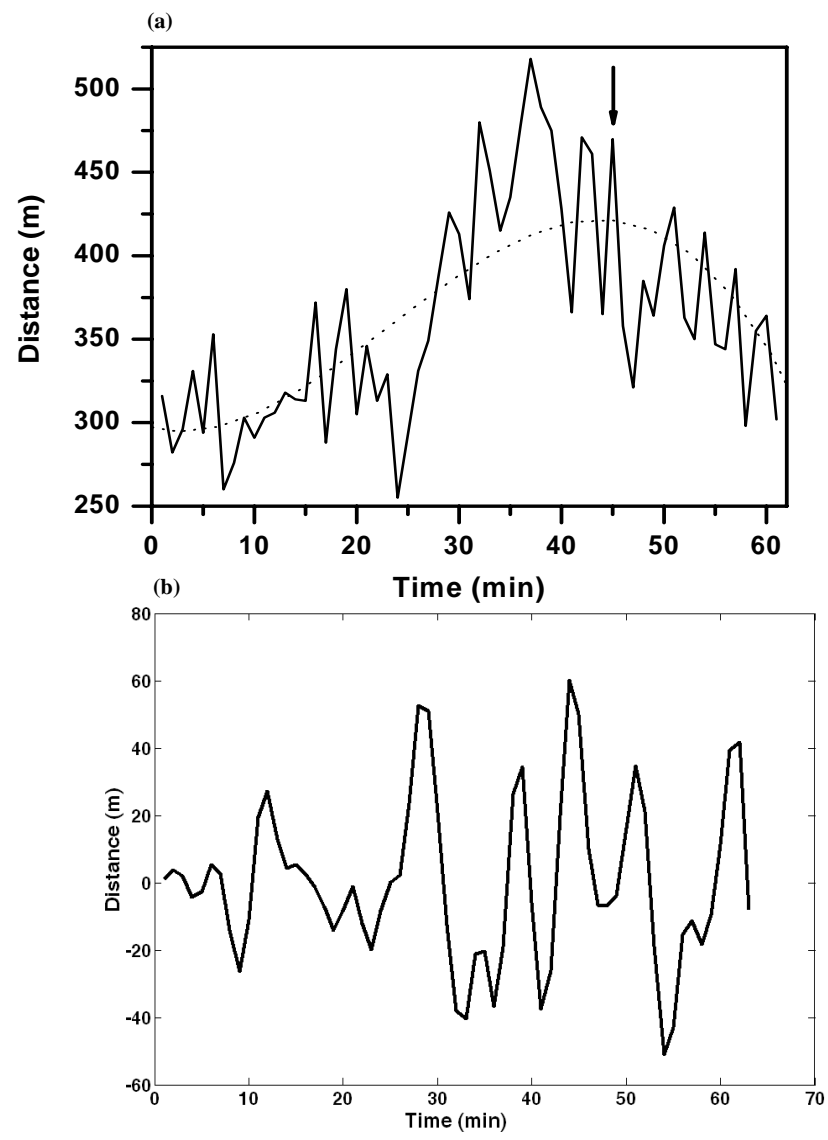

Fig. 2. (a) Time profile of the vertical distance traversed by the balloon in each successive minute computed directly from the radar tracking data as observed on 2 September 2002. The dashed line shows 3rd order polynomial fit. The arrow in the figure represents the altitude of cold point tropopause (b) The detrended and highpass filtered data of panel (a) (see text).

profile of balloon's ascent rate on the above mentioned date. The oscillatory nature of the ascent rate is readily observed form this figure. Figure $2 \mathrm{a}$ shows the time series of the vertical distance traversed by the balloon in each successive minute, computed directly from the radar tracking data. The dotted line in Fig. 2a represents third order polynomial fit as a probable trend representing the secular balloon motion. The polynomial fit is subtracted from the original time series to get the fluctuations in the distance traversed by the balloon. The resulting profile clearly brings out significant fluctuations in the ascent rate regime. This time series data is used for further analysis. It is a general observation from several data sets we have analysed that a large Brunt-Väisälä period over a significant altitude range of, say, $\sim 1 \mathrm{~km}$ or more, invariably has one or more unstable layers embedded within. Where the stratification is unstable, $\mathrm{N}^{2}$ goes negative, making Brunt-Väisälä period imaginary. Keeping this in view, the de-trended data are filtered using high pass filter having a cut off at $30 \mathrm{~min}$ and is shown in Fig. $2 \mathrm{~b}$.

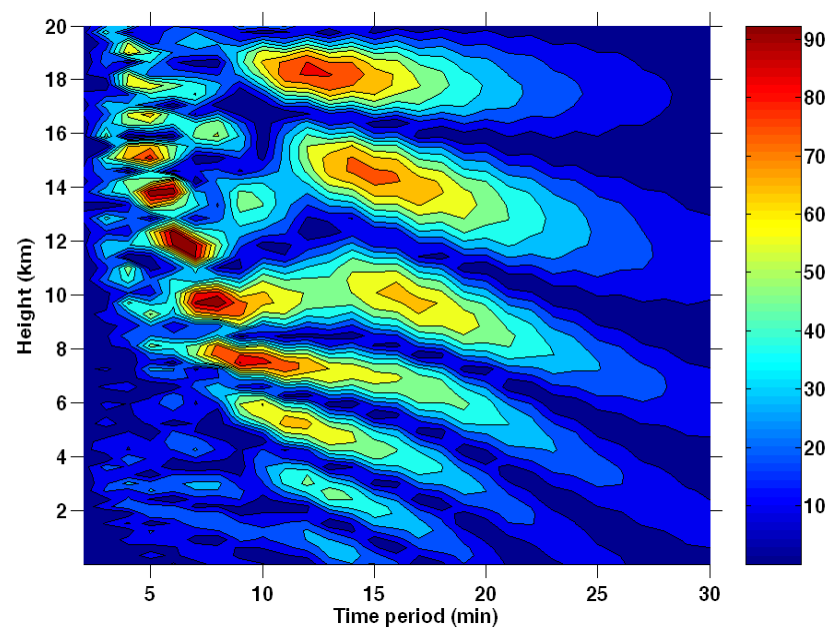

Fig. 3. The CWT amplitude spectra of time series of distance traversed by the balloon in each successive minute.

Now, the time series data shown in Fig. $2 b$ is subjected to the CWT and the corresponding amplitude spectrum is shown in Fig. 3. The $x$-axis in this figure shows the time period and y-axis shows the height. Alternating positive and negative maxima are clearly observed, and it is now a straightforward process to extract the dominant oscillations at each height. Following the procedure adopted by Rottger (1986) and Revathi et al. (1996), we take the Brunt-Väisälä period as corresponding to the amplitude gradient maxima rather than the amplitude maxima. It has been shown that the Brunt-Väisälä frequency will have a sharp falling edge, rather than a smooth transition towards the higher frequency side. This aspect is demonstrated in Fig. 4a and b. Figure $4 \mathrm{a}$ depicts the amplitude spectra at $18 \mathrm{~km}$ height region, which shows a peak at $15 \mathrm{~min}$ period. On the other hand, Fig. 4b, which plots the amplitude gradients, shows a dominant peak at $5 \mathrm{~min}$ period, a value characteristic of very stable stratospheric layers. From these two figures it can be seen that the amplitude gradients give more realistic measure of Brunt-Väisälä period than amplitude maxima. By adopting this procedure, the Brunt-Väisälä period at each height is identified. Figure 5a and $\mathrm{b}$ shows the height profiles of Brunt-Väisälä period on 2 and 12 September 2002, respectively. Each figure shows a profile derived from the present analysis and another corresponding to the Brunt-Väisälä period profile derived from the direct temperature observations using Eq. (1). Both the profiles are smoothed using 5-point running average. It should be noted that the smoothing of the data effectively reduces the vertical resolution of the profile. The smoothing is done in order to reduce some of the spikes observed in the Brunt-Väisälä period profiles. It is interesting to observe a good agreement between the two profiles. The estimated RMS differences given in the plots ensure the applicability of the present method. The retrieved dominant periods are around $10-15 \mathrm{~min}$ in the troposphere and 5-6 $\mathrm{min}$ in 
(a)

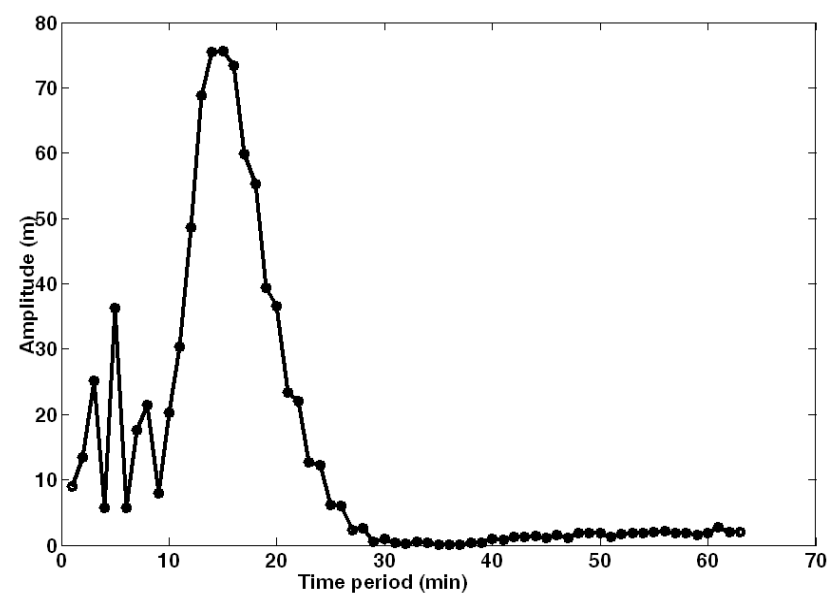

(b)

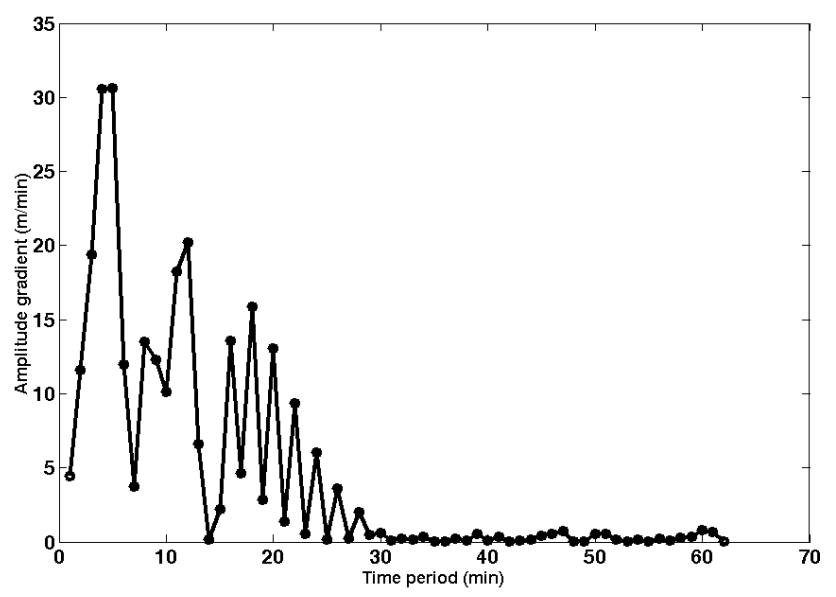

Fig. 4. (a) Amplitude spectra at $18 \mathrm{~km}$ height region and (b) same as (a) but for amplitude gradient.

the lower stratosphere. Earlier studies by Kitchen and Shutts (1990) reported that the wave period experienced by the ascending balloon is often close to the average Brunt-Väisälä period; the present study using the wavelet analysis proves this point beyond any doubt, and also provides the height profile of Brunt-Väisälä period, which has many practical applications. This comparison gives enough confidence to proceed further to derive Brunt-Väisälä period profile where simultaneous temperature measurements are not available, as from rawinsonde ascents at Shriharikota for example. That is what Fig. 6a shows for 4 September 2002 over shriharikota. As expected, this figure readily reveals the consistency of retrieved Brunt-Väisälä periods by showing that the stratosphere is more stable than the troposphere, and that the corresponding Brunt-Väisälä periods have the expected values.

We now proceed to derive the altitude variation of temperature from the Brunt-Väisälä period profile. The BruntVäisälä period profile is transformed to $\mathrm{N}^{2}$ profile and then (a)

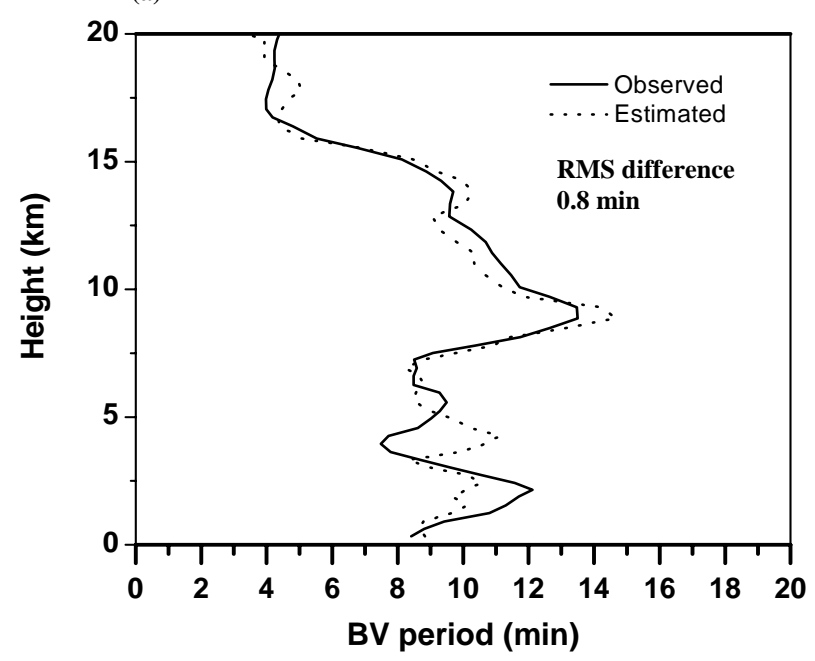

(b)

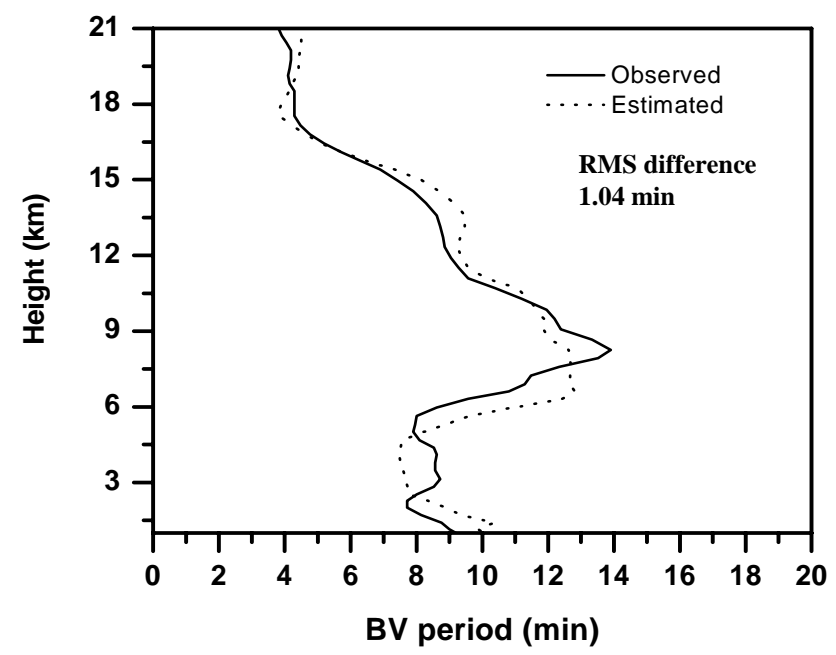

Fig. 5. Height profiles of Brunt-Väisälä period derived using wavelet analysis (dashed) and temperature sounding (solid) on (a) 2 September 2002 and (b) 12 September 2002 at Chennai.

it is a straightforward task to obtain temperature from the $\mathrm{N}^{2}$ profile. Integrating Eq. (2) we get

$$
T_{z}=\frac{1}{I_{z}}\left[I_{z_{o}} T_{o}-\Gamma \int_{z_{o}}^{z} I_{z} d z\right]
$$

with

$$
I_{z}=\exp \left[-\int_{z_{0}}^{z}\left(\frac{N^{2}}{g}\right) d z\right]
$$

where $T_{o}$ is the temperature at reference level $z_{o}$, surface value in our case. The sensitivity of the present method of retrieving temperature to the reference level temperature and associated errors were discussed in detail by Revathy et al. (1998). We use rawinsonde data from Shriharikota at 09:00 UT on 4 September 2002 for which the retrieved 
(a)

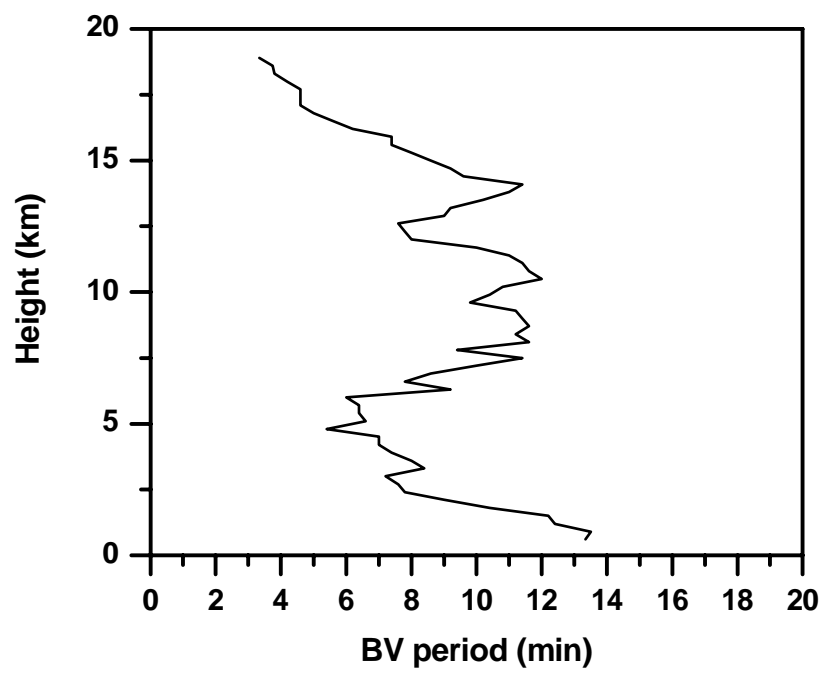

(b)

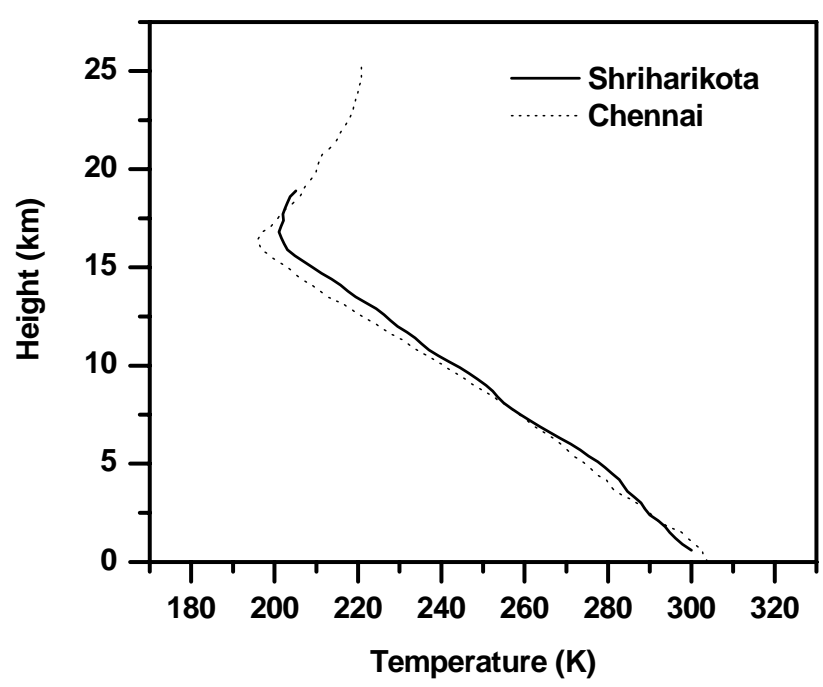

Fig. 6. Height profiles of (a) Brunt-Väisälä period and (b) temperature on 4 September 2002 over Shriharikota (09:00 UT). The dotted line represents temperature measured by the radiosonde at Chennai (12:00 UT), $100 \mathrm{~km}$ to the south, and the solid line represents temperature estimated from Eq. (3) by using $\mathrm{N}^{2}$ profile computed from present method.

Brunt-Väisälä period profile is shown in Fig. 6a. Figure 6b shows the resulting temperature profile (solid line) estimated from Eq. (3). The dotted line represents temperature measured by the radiosonde from Chennai at 12:00 UT. Even though there are some discrepancies in the profiles, which can be partly attributed to spatial separation of $100 \mathrm{~km}$ between the two stations, the overall agreement is reasonably good. Thus, the present method provides an unique opportunity to derive $\mathrm{N}^{2}$ profile and subsequently the temperature profiles using balloon ascent rates, which will find its application in rawinsonde ascents.

\section{Summary}

It is expected that the ascent of the balloon should respond to $\mathrm{N}$ in a stably stratified atmosphere. The altitude profile of radiosonde ascent rate is indeed observed to modulate with the Brunt-Väisälä frequency $(N)$. A wavelet approach is employed to estimate the vertical profile of Brunt-Väisälä period from the balloon ascent rate profile. The altitude profile of the Brunt-Väisälä period estimated from the present approach is compared with the profile computed from the temperature variation obtained from the same radiosonde. The agreement appears to be good. It has been demonstrated that the present method can be useful in determining the stability parameter where only wind measurements are carried out using rawinsondes. We have also derived the temperature profile by inverting $\mathrm{N}^{2}$ profile derived from the present method. The comparison between retrieved and measured temperature profiles shows reasonably good agreement. In the current scenario the agreements will significantly improve if one settles for coarser height resolution. Given that various factors associated with the ascent rate variability of a radiosonde, many of them known to be second order effects, and many that can only be qualitatively estimated, these results are perhaps nearly as good as it is possible to arrive at. This however does not preclude further work that is currently in progress.

Acknowledgements. Topical Editor F. D'Andrea thanks two anonymous referees for their help in evaluating this paper.

\section{References}

Chakrabarty, D. K., Shah, N. C., Pandya, K. V., and Peshin, S. K.: Long-term trend of tropopause over New Delhi and Thiruvananthapuram, Geophys. Res. Lett., 27, 2181-2184, 2000.

Daubechies, I.: Ten Lectures on Wavelets, Society for Industrial and Applied Mathematics, 357 pp, 1992.

De Mandel, R. E. and Krivo, S. J.: Radar/balloon measurement of vertical air motions between the surface and $15 \mathrm{~km}$, J. Appl. Meteorol., 10, 313-319, 1971.

De Mandel, R. E. and Scoggins, J. R.: Mesoscale wave motions as revealed by improved wind profile measurements, J. Appl. Meteorol., 6, 617-620, 1967.

Ecklund, W. L., Balsley, B. B., Carter, D. A., Riddle, A. C., Crochet, M., and Garello, R.: Observations of vertical motions in the troposphere and lower stratosphere using three closely spaced ST radars, Radio Sci., 20, 1196-1206,1985.

Farge, M.: Wavelet transforms and their applications to turbulence, Ann. Rev. Fluid Mech., 24, 395-457, 1992.

Foufoula-Georgiou, E. and Kumar, P. (Eds.): Wavelets in Geophysics, Academic Press, New York, 373 pp., 1995.

Holton, J. R.: An introduction to dynamic meteorology, Academic Press, New York, 511 pp., 1992.

Hooper, D. A., Arvelius, J., and Stebel, K.: Retrieval of atmospheric static stability from MST radar return signal power, Ann. Geophys., 22, 3781-3788, 2004, http://www.ann-geophys.net/22/3781/2004/. 
Kitchen, M. and Shutts, G. J.: Radiosonde observations of largeamplitude gravity waves in the lower and middle stratosphere, J. Geophys. Res., 95(D12), 20451-20455, 1990.

Lalas, D. P. and Einaudi, F.: Tropospheric gravity waves: their detection by and influence on rawinsonde balloon data, Q. J. Roy. Meteorol. Soc., 106, 855-864, 1980.

Massman, W. J.: On the nature of vertical oscillations of constant volume balloons, J. Appl. Meteorol., 17, 1351-1356, 1978.

Murrow, H. N. and Henry, R. M.: Self induced balloon motions, J. Appl. Meteorol., 4, 131-138, 1965.

Reid, S. J.: An observational study of lee waves using radiosonde data, Tellus, 24, 593-596, 1972.

Revathy, K., Prabhakaran Nair, S. R., and Krishna Murthy, B. V.: Deduction of temperature profile from MST radar observations of vertical wind, Geophys. Res. Lett., 23, 285-288, 1996.

Revathy, K., Nair, S. R. P., and Krishna Murthy, B. V.: Estimation of error in the determination of temperature using MST radar, Indian J Radio Space Phys., 27, 241-243, 1998.
Rottger, J.: Structure and dynamics of the stratosphere and mesosphere revealed by VHF radar investigations Pure Appl. Geophys., 118, 494-527, 1980.

Rottger, J.: Determination of Brunt-Väisälä frequency from vertical velocity spectra, MAP hand book, 20, 168-172, 1986.

Salby, M. L.: Fundamentals of atmospheric physics, Academic Press, New York, 627 pp., 1996.

Schmidlin, F. J.: WMO international radiosonde comparison phase II, 1985, WMO Instrum. Obs. Methods Rep., 29, WMO/TD 312, 113 pp., World Meteorol. Org., Geneva, Switzerland, 1988.

Vincent, R. A. and Alexander, M. J.: Gravity waves in the tropical lower stratosphere: An observational study of seasonal and interannual variability, J. Geophys. Res., 105, 17971-17982, 2000.

Wang, L. and Geller, M. A.: Morphology of gravity wave energy as observed from four years (1998-2001) of high vertical resolution U.S. radiosonde data, J. Geophys. Res., 108(D16), 4489, doi:10.1029/2002JD002786, 2003.

Weinstein, A. I., Reiter, E. R., and Scoggins, J. R.: Mesoscale structure of 11-20 km winds, J. Appl. Meteorol., 5, 49-57, 1966. 